\title{
Los juegos de rol y el rendimiento académico en los cursos de periodismo
}

\author{
Llamil Vásquez Valencia \\ Universidad Nacional Mayor de San Marcos \\ llamilv@yahoo.com
}

\begin{abstract}
RESUMEN
La investigación consistió en determinar el tipo de relación que existe entre el método didáctico de los juegos de rol y el rendimiento académico de los alumnos de la especialidad de periodismo de la EAP de Comunicación Social de la UNMSM en el periodo 2017-II.

El tipo de investigación es descriptivo correlacional causal. El diseño es no experimental descriptivo correlacional causal, cuantitativo y de corte transversal. La conclusión es que existe una relación directa y significativa de $\mathrm{r}=0.633$ entre los juegos de rol y el rendimiento académico de los estudiantes.

Los hallazgos recomiendan que se afiancen más capacitaciones pedagógicas a los profesores de la especialidad de periodismo de la EAP de Comunicación Social de la UNMSM para sistematizar y evaluar la aplicación de los juegos de rol con el fin de incidir favorablemente en el rendimiento académico de los estudiantes y en su desarrollo como futuros profesionales del periodismo.
\end{abstract}

Palabras clave: juegos de rol, rendimiento académico, rol del profesor, selección de participantes, reglas de juego.

\section{Role playing and academic performance in journalism courses}

\begin{abstract}
The investigation consisted of determining the type of relationship that exists between the didactic method of the role games and the academic performance of the students of the specialty of journalism of the EAP of Social Communication at the UNMSM in the period 2017-II.

The type of research is descriptive causal correlational. The design is non-experimental descriptive correlational causal, quantitative and cross-sectional. The conclusion is that there is a direct and significant relationship of $\mathrm{r}=0.633$ between role plays and students' academic performance.

The findings recommend that more pedagogical training be given to the professors of the Journalism specialty of the EAP of Social Communication at the UNMSM to systematize and evaluate the application of role-playing games in order to favorably affect the academic performance of the students and in their development as future journalism professionals.
\end{abstract}

KEYwORDs: role plays, academic performance, teacher rol, selection of participants, game rules. 


\section{Introducción}

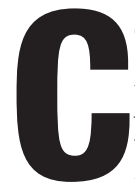
on frecuencia los estudiantes o egresados de la carrera de Comunicación Social de la mayoría de universidades del país tienen problemas para redactar su primera nota informativa y la primera observación que reciben generalmente del editor es que no han tomado en cuenta el contexto de la noticia. En ese sentido, investigaciones anteriores sugieren que el método didáctico de los juegos de rol elevan el rendimiento académico de los estudiantes en todos los aspectos (Yurani, 2014; Forero y Loaiza, 2013; Cáceres, 2012; Gaete-Quezada, 2011; Martínez, 2009; Vera-Mostacero, 2007).

Por ejemplo, Gaete-Quezada (2011), en su investigación El juego de roles como estrategia de evaluación de aprendizajes universitarios, tuvo como objetivo determinar la relación práctica del uso del juego de rol como estrategia didáctica para obtener aprendizaje significativo en la formación de estudiantes universitarios de la Universidad de Antofagasta, Chile. La muestra estuvo constituida por 250 estudiantes de tercer año de las carreras de Administración Pública, Administración de Empresas y Trabajo Social. La metodología fue descriptivo correlacional, diseño no experimental. Los resultados mostraron una mejora evidente en el rendimiento académico de los estudiantes, en comparación con la evaluación tradicional de los contenidos de la asignatura observada. Además, se aportaron elementos metodológicos para replicar la experiencia.

Esta investigación demuestra la importancia del método didáctico de los juegos de rol en la mejora de los aprendizajes en los estudiantes universitarios y el consiguiente rendimiento académico en varias carreras enmarcadas en el ámbito de las ciencias sociales. Sin embargo, no describe sus características particulares en una carrera específica.

El periodismo es una carrera compleja debido a su carácter interdisciplinario, por lo que es necesaria la aplicación del método didáctico de los juegos de rol. Gaete-Quezada (2011) señala que la formación de profesionales universitarios es un proceso complejo, que requiere de una serie de condiciones materiales, técnicas y humanas, necesarias para adquirir competencias laborales adecuadas para insertarse en el mundo laboral, con el fin de desempeñar actividades específicas que demandarán un dominio concep- tual y práctico de una serie de conocimientos y técnicas propias de su respectiva especialidad profesional.

El método didáctico de los juegos de rol ofrece variadas posibilidades para el aprendizaje de los conocimientos y técnicas del periodismo debido a su carácter vivencial o experiencial. Birkenbihl (2008) resalta que los juegos de rol tienen fines eminentemente pedagógicos pues los estudiantes reciben instrucciones sobre cómo comportarse en una aventura de aprendizaje que a la larga se convierte en una historia.

¿Cuáles son las ventajas de los juegos de rol? En primer lugar permite que el grupo discuta de forma acalorada, incluso a veces violenta. A menudo se producen opiniones muy dispares sobre si un actor debe ser censurado por su actitud, cuáles son los motivos que provocan determinada conducta y cuál sería el mejor camino para corregir o dominar una situación complicada. (Birkenbihl, 2008, p. 201).

De esta manera, Birkenbihl (2008) destaca que el estudiante extrae las siguientes ideas: Se acostumbra a no formarse un juicio a la ligera sobre las personas o su comportamiento al trabajar con ellas en grupos; comprende que no existe nunca una solución «absolutamente» correcta para los problemas; observa que la misma situación es percibida de modo totalmente distinto por las personas afectadas; comprueba que no son justificados muchos prejuicios, por ejemplo, respecto a los supuestos privilegios del director del juego o cualquier otro participante que tenga una apariencia peculiar; aprende a reconocer ampliamente el valor que tienen las discusiones con sus compañeros; y aprende a deshacerse de conceptos idealistas más o menos ajenos a la realidad, porque se ve forzado a establecer sus consideraciones dentro de ciertos límites que vienen determinados por diversos factores.

Como se advierte, los estudiantes de periodismo pueden aprender a solucionar problemas a través de una discusión horizontal y formarse de manera significativa si es que encuentran una utilidad práctica a los conocimientos que adquieren.

El juego de rol en el que se tratan problemas de grupo revela otra de las aptitudes menos apreciadas en la práctica cotidiana: saber escuchar. El que di- 
rige una entrevista de modo autoritario, sin pedir sugerencia a los participantes, nunca conseguirá una buena solución a los problemas. (Birkenbihl, 2008, p. 205).

De esta manera, el estudiante encuentra informaciones relevantes que serían vistas como vanas en una situación de aprendizaje teórico y eminentemente bibliográfico. En otras palabras, los conocimientos son percibidos como significativos cuando están vinculados con la experiencia.

El éxito del juego de rol se basa [...] en que los participantes defiendan su propia opinión. Dicho de otra manera, cuando, por ejemplo, un participante tiene que desempeñar el papel de una persona, tendrá que actuar tal y como lo haría el jefe de personal de verdad y no cómo se imagina que actuaría un jefe de personal. Antes de empezar el juego hay que dejar claro este importante aspecto a todos los participantes. (Birkenbihl, 2008, p. 205).

En conclusión podemos señalar que el método didáctico de los juegos de rol es una dramatización de un aspecto de la realidad que establece el profesor en el aula con el fin de que los alumnos adquieran conocimientos al interpretar personajes enmarcados en un conjunto de reglas. Esta técnica se basa en una teoría multidisciplinaria enmarcada en la corriente pedagógica del constructivismo y es considerada parte de la metodología activa y de los juegos didácticos derivados de esta.

En la carrera de periodismo los métodos tradicionales de enseńanza son limitados cuando se imponen conocimientos a través de lenguajes que no son necesariamente significativos para el alumno y que impiden generalmente el desarrollo de su curiosidad y los intereses propios de su nivel de desarrollo cognitivo.

El método didáctico de los juegos de rol, como dramatización interactiva realizada por el profesor y sus alumnos, puede ser medido en base a las calificaciones de las habilidades desarrolladas por los personajes para solucionar problemas en un escenario real donde se siguen reglas generales. El objetivo es contar una historia que puede ser real y es interactivo porque los estudiantes tienen la capacidad de modificar sus acciones con el fin de salir airosos de un conflicto y aprender sobre la experiencia (Amézquita y Moreno, 2001).

\section{Características del método didáctico de los juegos de rol}

En los juegos de rol educativo se distinguen dos tipos de participantes: el director del juego y los participantes. Los alumnos interpretan a personajes que trabajan de manera conjunta para alcanzar una meta establecida en la trama del juego. Según Amézquita y Moreno (200l), el director, generalmente interpretado por el docente, es el árbitro y desempeña las siguientes tareas:

1) La descripción del contexto o el escenario.

2) El control y análisis de los sujetos que son parte del contexto y con quienes van a interactuar los participantes.

3) La determinación del éxito de las acciones de los alumnos a través de la interpretación de las reglas.

Birkenbihl (2008) indica que «una vez distribuidos los papeles, hay que dejar algún tiempo antes de comenzar el juego para que los actores se familiaricen con su papel» (pag.205), es decir identificarse con el personaje que van a interpretar.

No debe ocurrir que un actor tenga que mirar sus apuntes durante el juego porque no se acuerda de los hechos que constituyen la base del problema. Esto interrumpe el flujo de la acción y evidencia que solo es un juego. (Birkenbihl, 2008, p. 205)

El método didáctico de los juegos de rol se apoya en la interacción entre el director del juego y los jugadores. Mientras el director del juego observa y supervisa qué hacen los jugadores y cómo lo hacen; los jugadores desempeñan sus roles en el transcurso del juego. El director determina, entonces, qué tanto éxito han tenido basándose en las reglas (Amézquita y Moreno, 2001).

\section{Fases de los juegos de rol en la escuela}

Los profesores norteamericanos Fannie y George Shaftel diseñaron por primera vez en 1967 un modelo de enseńanza escolar basado en los juegos de rol, la cual requería que los alumnos representen conflictos para aprender a asumir los roles de otros y observar 
al mismo tiempo la conducta social de su entorno (Joyce, B. y Weil, M., 2009).

Los esposos Shaftel determinaron que el desarrollo del juego de roles debe pasar por nueve fases, para enriquecer el aspecto central de la actividad de aprendizaje:

1) Preparación de los grupos: El profesor plantea un problema con ejemplos cotidianos, para provocar el interés de los alumnos y crear un clima de confianza, a fin de que los alumnos puedan exponer todas sus ideas, sentimientos y conductas con libertad.

2) Selección de los participantes: Profesor y alumnos elaboran el motor de cada personaje (quién es, cómo es, qué hace, qué siente), para que los mismos alumnos elijan los roles que quieren representar.

3) Ambientación del escenario: Se hace un esbozo de la situación y se orienta una línea de acción.

4) Preparación de los observadores: Los observadores deben estar muy implicados en la representación, y para tal efecto se sugiere que el profesor les asigne ciertas tareas (evaluar el realismo, definir los personajes, determinar la eficacia de la representación y las alternativas de acción).

5) Actuación: Los jugadores deben vivir la situación espontáneamente, respondiendo a sus motivaciones personales. El actor no debe manifestar respuestas pensadas a priori, sino que se debe expresar según lo que suceda en la situación concreta que experimenta.

6) Discusión y evaluación: La discusión y evaluación sobre la representación debe ser espontánea, y eso sucederá en la medida que los actores y observadores estén verdaderamente comprometidos. El diálogo debe centrarse en «las consecuencias de la acción y en las motivaciones del actor».

7) Nueva actuación: Las representaciones, enriquecidas por las discusiones, deben explorar nuevas posibilidades de acción e intercambio de papeles.

8) Discusión y evaluación: Los alumnos deben plantear conjuntamente una solución al problema, frente a lo cual el profesor hará hincapié en el realismo de la conclusión, examinando si esa opción puede darse en la vida real.

9) Compartir experiencias y generalizar: El fin es relacionar los problemas (representados, discutidos y evaluados) con la experiencia cotidiana, para formular principios de comportamiento y su aplicación a situaciones reales (Joyce, B. y Weil, M., 2009, p. 98).

\section{Adaptación universitaria del modelo Shaftel en la UNMSM}

En nuestra investigación sobre los juegos de rol educativo empleados en la especialidad de Periodismo de la EAP de Comunicación Social de la UNMSM, tomando como guía el diseño de los esposos Shaftel, se percibieron cuatro dimensiones que son:

1) El rol del profesor en la motivación.

2) La selección de los participantes.

3) Las reglas de juego.

4) La actuación-evaluación.

\section{El rol del profesor}

La actuación del docente es importante, porque es el director del método didáctico de los juegos de rol. Su tarea es motivar y fomentar la participación democrática de los alumnos en la identificación de los problemas y crear un clima de confianza para la libre expresión de las ideas. Este es el rol que han asumido en general los profesores de la especialidad de Periodismo en la Escuela Profesional de Comunicación Social de la UNMSM.

En esta fase del proceso el docente tiene que explicar a los alumnos el concepto de rol de modo que el alumno se piense a sí mismo como actor e identifique a sus compañeros como tales. En este caso, el profesor asume el papel de director de un diario y los alumnos tienen la misión de jugar a ser periodistas especializados en diferentes campos de interés noticioso, denominados en la jerga profesional como secciones.

Joyce, B. y Weil, M. (2009) afirman que:

El concepto de rol es uno de los fundamentos teóricos esenciales del modelo de juego de roles. También es un muy importante objetivo. Debemos enseñar a los alumnos a utilizar este concepto, a reconocer roles diferentes, a pensar en la propia conducta y en la de otros en términos de roles (p. 92).

Hecho esto, el profesor explica la naturaleza y características de la labor de cada sección en un periódico. Aquí, los estudiantes tendrán la libertad de partici- 
par en el reconocimiento del escenario, pues este ya está establecido de antemano y es el mismo contexto social que rodea al alumno en cuanto a que observador de la realidad como una entidad generadora de noticias.

Como vemos, el docente tiene la misión de preparar y animar a los estudiantes a participar en esta aventura de aprender. Joyce, B. y Weil, M. (2009), basándose en el modelo de los Shaftel, mencionan que el rol del profesor en esta fase consiste en presentar un problema que debe suscitar el interés de todos los alumnos al tiempo que crea un clima de confianza.

El docente, en su rol de director del periódico, se encarga luego de explicar las tareas o comisiones periodísticas que realizará cada grupo y los avances que reportarán semanalmente hasta el fin del curso. No se descarta que un tema elegido pueda cambiarse por otro cuando se trata de una noticia que no tiene continuidad en el tiempo.

Los hechos de interés público preferidos en la sección política son los casos de corrupción como el caso Odebrecht o Lavajato, los cuales pueden actualizarse cada semana y tienen una duración que va más allá de un ciclo académico. A los participantes se les indica que pueden usar como fuentes portales periodísticos de investigación acreditados como IDL-Reporteros y Ojo Público, además de las páginas de medios como El Comercio, La República, RPP y Canal N, para estar actualizados.

Para efectos de contar con fuentes propias se puede entrevistar a conocidos constitucionalistas o juristas. Estos especialistas pueden orientar a los estudiantes en los aspectos jurídicos y procesales de cada caso relacionado a Odebrecht: por ejemplo, evaluar la situación legal del expresidente Alan García en el caso de la Matanza de El Frontón.

La labor del docente será supervisar semanalmente el avance de los estudiantes y orientar en el tratamiento de la información con el fin de que pueda ser publicable en una revista impresa o en una página periodística de Facebook enlazada con otras redes sociales como el twitter.

El tratamiento de la información está relacionado con el derecho universal a la libertad de expresión y los juicios de valor de los participantes tienen que ir en consonancia con la ética periodística.
De esta manera, el rol del docente se apoyará en: Fomentar la participación de los alumnos en cada clase; establecer el escenario periodístico; recomendar las tareas de cada sección periodística; orientar en un eventual replanteamiento de las tareas periodísticas; y crear un clima de confianza para favorecer la libre expresión de las ideas.

\section{Selección de los participantes}

El rol del docente en la selección de los participantes de cada grupo con sus respectivos líderes es el más difícil, pues debe preparar la aventura de aprender a conciencia y dominar todos los detalles de la puesta en marcha de un periódico de calidad, antes de plantearlo a los alumnos que oficiarán de periodistas.

Es importante destacar que no todos los alumnos reaccionan de la misma manera ante una propuesta, por lo que el director de juego debe tener preparadas algunas alternativas de solución a cada objeción. El profesor debe tomar en cuenta la elección de los alumnos respecto a qué sección o grupo pertenecer, ya sea locales, policiales, política, judiciales, economía, espectáculo o deportes. Joyce, B. y Weil, M. (2009) explican que «el docente y los alumnos describen los diversos personajes (cómo son, cómo piensan y qué podrían hacer)» (p. 96), para incentivar su identificación con determinado rol.

El liderazgo en cada sección debe ser consensuado para evitar enfrentamientos y disensiones posteriores. De este modo, la elección del jefe de cada sección será consecuencia de la libre elección de los participantes de cada sección periodística.

Conformados los grupos, los alumnos pueden consultar con el profesor las medidas a tomar con los participantes que no cumplan con entregar sus informes semanales sobre un tema específico o un tema grupal. En este punto es necesario el arbitrio constante del profesor, para evitar desacuerdos que no llegan a ningún lado o posibles conflictos entre los participantes.

De este modo se logra: la identificación con los roles; la conformación democrática de los grupos; una elección adecuada de líderes; el establecimiento consensuado de una sanción para los infractores; y un arbitrio en la elección de los roles.

3. Las reglas de juego 
Con la aplicación de los juegos de rol en los cursos de la especialidad de periodismo se produce un acercamiento al trabajo real de una sala de redacción periodística, cuyo desarrollo puede llevarse perfectamente dentro y fuera del aula con el concurso de los alumnos, los cuales asumen el rol de periodistas para elaborar sus informes en base a hechos reales.

Amézquita y Moreno (2001) consideran que las reglas son una buena guía, pero no siempre son aplicadas de manera estricta, sino que cada docente es libre de moldearlas de acuerdo a las maneras de jugar de los grupos.

El docente de un curso de periodismo oficia de director y crea las reglas de una "historia" que los alumnos van moldeando y retocando a partir de las acciones que realizan. Para ello, el profesor suele usar las reglas basadas en una bibliografía especializada, las cuales varían según los objetivos de aprendizaje y de la especialidad.

En este juego, el profesor tiene la responsabilidad de establecer las pautas del tipo de publicación elegida (semanal o mensuario), escoger en última instancia el tipo de soporte (digital o impreso) y constituir las secciones que puedan desarrollarse de acuerdo a la disponibilidad y cantidad de alumnos.

Las reglas se basan en las técnicas de redacción periodística, en especial el de la crónica. Los alumnos utilizarán el género de la crónica, debido a que esta se apoya en la interpretación y la descripción para su publicación en cualquier medio. La calidad de este tipo de información periodística se basa en la valoración de las noticias y las opiniones de especialistas.

El docente-director de juego es también el encargado de supervisar que la línea editorial del curso concuerde con la deontología profesional del periodista, el cual se basa en el derecho universal a la libertad de expresión y a la libertad de información, así como tener en cuenta los límites que esas libertades pueden tener.

En este caso, la deontología toma como guía la Declaración de principios de la Federación Internacional de Periodistas (FIP) sobre la conducta de los periodistas (aprobada en 1954 por el Congreso de la FIP), el cual se ha vuelto en el principio rector de los periodistas y de sus sindicatos para aplicar ética y calidad al periodismo en el mundo.

Las reglas de juego periodístico incluyen, también el uso necesario de los apuntes y la pertinencia del manejo de las grabadoras, los imprevistos que se puedan presentar y su preparación ante estos. Las recomendaciones, ante este tema, pueden parecer una nimiedad, pero la experiencia demostrará su importancia.

Los participantes deben conocer cómo se elabora una agenda periodística diaria y semanal, además de la necesidad del reporte constante de su cumplimiento, para detectar problemas para su solución. Es conveniente aclarar que la revisión de las tareas nos acerca a la fase de actuación-evaluación en su conjunto.

Las reglas de juego, entonces, están conformadas por: las técnicas de redacción; las pautas de conducta; el uso de apuntes y grabadoras; la elaboración de la agenda periodística; y un reporte de los avances periodísticos.

\section{Actuación-evaluación}

Debe remarcarse, ante todo, que un juego de rol se basa en la interpretación y en la capacidad de improvisación del profesor y de los alumnos, por lo que restringir el avance de la partida a una serie de reglas escritas es eliminar el mayor punto de diversión. En estos juegos experienciales se produce también una actuación-evaluación constante entre todos los participantes.

En la actuación el objetivo es constituir los hechos y los roles, a fin de evaluarlos, para volver a representarlos en el transcurso de la representación. Los participantes deben expresar todo lo que le suceda en cada situación concreta que experimente. La actuación-evaluación se repite tantas veces mientras dure el curso,

Las actuaciones-evaluaciones permitirán solucionar problemas y mejorar el desempeño de los participantes con su consecuente enriquecimiento de los aprendizajes y el rendimiento académico. Joyce, B. y Weil, M. (2009) afirman que:

En la representación los actores asumen los roles y «viven» la situación espontáneamente, respondiendo uno al otro en forma realista. [...] Esta incertidumbre es parte de la vida y a la vez forma parte de sentir el rol. [...] La actividad se alterna entre la discusión y la actuación. (pp. 97-98)

En los cursos de especialidad de periodismo, el docente y los alumnos evaluarán el grado de importan- 
cia periodística de los datos que han procesado en base a los criterios de actualidad, novedad, curiosidad, proximidad, novedad, carácter de la fuente, exclusividad, conflicto, interés humano, etc.

Los alumnos, en su rol de periodistas, aprenderán a comprobar la veracidad de los datos cuando se topen con noticias falsas o rumores que tanto abundan actualmente en internet y en la sociedad en su conjunto.

Además, tanto el director del juego y los periodistas revisan y corrigen constantemente el estilo de los informes semanales. El objetivo no es solo cumplir con escribir un informe con un estilo aceptable, sino de lograr afinar un estilo propio y desarrollar la creatividad en el enfoque y la escritura.

De esta manera, los participantes se involucran en el talante crítico y autocrítico de la labor periodística en sus dos vertientes, que son como las dos caras de una misma moneda: una búsqueda constante de la ejecución correcta de la forma y el contenido de los informes.

En consecuencia, el objetivo es que los participantes se acostumbren a realizar y desarrollar una adecuada: evaluación de los datos periodísticos; verificación de datos; supervisión de la redacción periodística; analizar la creatividad estilística; y brindar sugerencias para mejorar el desempeño periodístico.

\section{El rendimiento académico}

El método didáctico de los juegos de rol, que emplean los profesores en los cursos de la especialidad de periodismo de la EAP de Comunicación Social de la UNMSM, elevó el rendimiento académico de los alumnos en el semestre 2017-II. El informe de los promedios finales demostró que su carácter vivencial conecta a los estudiantes con su contexto produciéndose una mejora de sus competencias en los aspectos cognitivo, procedimental y actitudinal.

Según Hernández y Barraza (2013), el rendimiento académico de los estudiantes de nivel superior es la suma de diferentes y complejos factores que interactúan en la persona que aprende, y ha sido definido con un valor atribuido al logro del estudiante en las tareas académicas. El rendimiento, entonces, se mide mediante las calificaciones obtenidas, con una valoración cuantitativa, cuyos resultados muestran las materias ganadas o perdidas, la deserción y el grado de éxito académico.

Álvaro, M. et al. (1990) resalta la dificultad que estriba en conceptuar el rendimiento académico debido a que se encuentra determinado por múltiples dimensiones, entre las que destaca la social, educativa-institucional y económica. Sin embargo, reconoce que las medidas más utilizadas para el rendimiento académico han sido, por una parte, las calificaciones escolares y, por otra, las distintas pruebas objetivas o exámenes de rendimiento creados «ad hoc».

El rendimiento académico es indiscutiblemente un concepto multidimensional, en donde convergen distintas variables y distintas formas de medición. Dependiendo de las variables previamente seleccionadas por un modelo causal y de las medidas de rendimiento, se tendrán concepciones diversas sobre el tema, que conducirán a estrategias de análisis e interpretación determinadas. En este tipo de análisis se consideran, sobre todo, los componentes de contenido cognitivo, no contemplándose aspectos tales como las actitudes, el autoconcepto, la satisfacción, las expectativas, etc. Considerada esta limitación en la medida, el criterio más utilizado por los investigadores es el de emplear como posibles medidas predictoras del rendimiento, tanto las calificaciones escolares como distintas pruebas objetivas, precisamente para poder contrarrestar la diversidad de criterios de evaluación que, de hecho, contaminan los resultados reales en el aprendizaje de los alumnos. (Álvaro, M. et al., 1990, p. 24).

\section{A) La visión crítica como dimensión actitudinal}

El rol de un periodista es producir mensajes informativos que no pueden estar divorciados del análisis crítico de todo hecho de interés público (Van Dijk, 1997). En ese sentido, podríamos afirmar que un "periodista que no es crítico, no es periodista». Existe una frase bien conocida, que ha sido atribuida sin confirmar al escritor George Orwell, pero también ilustra lo que todo periodista sabe perfectamente: «Una noticia es aquello que alguien no quiere que se publique. El resto son relaciones públicas».

En ese sentido, es obvio que los juegos de rol periodístico también pueden utilizar los nuevos soportes tecnológicos, como Facebook, Twitter y You Tube, para que los estudiantes puedan transmitir 
noticias en vivo y evaluar su desempeño con la orientación del profesor.

Los portales periodísticos permiten, de otro lado, que los alumnos puedan evaluar si los mensajes que transmiten estos medios son confiables con la utilización del Análisis Crítico del Discurso (ACD) propuesto por Van Dijk (1997). En este caso, los estudiantes utilizan como fuentes a estos medios para producir noticias con una visión propia.

El profesor tiene la misión de orientar a los alumnos en la actividad inicial que supone el ACD para obtener los datos reales que sirvan como insumo de la producción de las noticias por parte de los alumnos.

El ACD los juegos de rol obliga al estudiante a desarrollar un juicio crítico en base a una lectura atenta de las editoriales de posiciones políticas e ideológicas distintas, si es que no contrapuestas, como $\mathrm{El}$ Comercio y La República, por citar un ejemplo.

Van Dijk (1991) señala que el punto de vista del periodista juega un papel importante en la producción de una noticia, pues esta no solo es escribir un texto periodístico en base a las declaraciones, las entrevistas, las reuniones, las conferencias de prensa, los debates parlamentarios, los juicios en los tribunales, etc.

Por su parte, Kapuscinski (2002), concluye que una cualidad del buen periodista es tener sintonía con los demás:

Creo que para ejercer el periodismo, ante todo, hay que ser un buen hombre o una buena mujer: buenos seres humanos. Las malas personas no pueden ser buenos periodistas. Si se es una buena persona, se puede intentar comprender a los demás, sus intenciones, su fe, sus intereses, sus dificultades, sus tragedias. Y convertirse, inmediatamente desde el primer momento, en parte de su destino. Es una cualidad que en psicología se denomina «empatía». Mediante la empatía se puede comprender el carácter del propio interlocutor y compartir de forma natural y sincera el destino y los problemas de los demás (...)

El verdadero periodismo es intencional, a saber: aquel que fija un objetivo y que intenta provocar algún tipo de cambio. No hay otro periodismo posible. Hablo, obviamente, del buen periodismo. Si leéis los escritos de los mejores periodistas-las obras de Mark Twain, de Ernest Hemingway, de Gabriel García Márquez-comprobaréis que se trata siempre de periodismo intencional. Están luchando por algo. Narran para alcanzar, para obtener algo. (pp. 38-39)

\section{B) La valoración periodistica como dimensión conceptual}

Van Dijk (1990) señala que los valores periodísticos reflejan los valores económicos, sociales e ideológicos de la sociedad dominada por grupos de poder, que buscan vender sus productos y el encubrimiento de hechos perpetrados por las élites que perjudican a la ciudadanía.

La consciencia de esta situación en la producción de las noticias permite que los alumnos asuman su papel como periodistas con responsabilidad, para no caer en la banalidad de convertir las noticias en un espectáculo vendible dejando de lado los principales problemas que aquejan a la ciudadanía.

De esta manera, el alumno verá potenciada su percepción de los hechos de interés público y su capacidad de investigación en beneficio de la sociedad. Con esta actitud el estudiante tendrá una crítica para producir noticias de manera óptima al tiempo que influirá de manera positiva en su rendimiento académico.

Van Dijk (1990) reconoce, también, la necesidad de emplear ciertos criterios de valoración periodística para reconocer si un hecho es una notica en un determinado contexto social. Estos criterios, conocidos por la mayoría de los periodistas como la novedad, la actualidad, la relevancia, la proximidad, entre otros, solo pueden aprendidos por los alumnos si asumen su rol de periodistas.

\section{C) Los juegos de rol y la dimensión procedimental}

El método didáctico de los juegos de rol puede potenciarse con la publicación de los mensajes periodísticos en blogs, páginas de Facebook y canales de You Tube, puesto que estos soportes cuentan con la ventaja de contar con una poderosa herramienta conocida como hipertexto.

Uno de los portales periodísticos más importantes en la actualidad es IDL-Reporteros por destapar el caso de "Los cuellos blancos del puerto" con la publicación de audios que comprometen a jueces en los presuntos delitos de criminalidad organizada, tráfico de influencias, cohecho pasivo propio y otros ilícitos en agravio del Estado, según la investigación fiscal. La labor de IDL-Reporteros es un ejemplo de 
como un portal periodístico puede tener éxito en el campo de la investigación periodística, tanto como lo puede tener un diario impreso, una radio o un programa dominical. El método de investigación periodística es el mismo en todos los casos, si bien es cierto que la forma de presentación de los mensajes cambia de acuerdo al soporte tecnológico.

El director de IDL-Reporteros, Gustavo Gorriti, es un reconocido periodista que se inició como investigador en la revista Caretas de la mano de su fallecido fundador y director Enrique Zileri. Fruto de su labor como investigador en dicho semanario, Gorriti ha publicado libros importantes como «Sendero» y «Calavera en negro». Es evidente que su visión crítica respecto a la corrupción en la política peruana es propia de la función de todo periodista que se precie como tal.

\section{Objetivos del estudio}

El objetivo general de la presente investigación fue establecer la relación entre el Método Didáctico de los Juegos de Rol y el Rendimiento Académico de los alumnos de la especialidad de periodismo de la EP de Comunicación Social de la UNMSM en el periodo 2017-II.

Los objetivos específicos fueron: 1- determinar la relación entre el Rol del Profesor y el Rendimiento Académico de los alumnos; 2-determinar la relación entre la Selección de los Participantes en el Juego de Rol y el Rendimiento Académico de los alumnos; 3-determinar la relación entre las Reglas de los Juegos de Rol y el Rendimiento Académico de los alumnos; 4- determinar la relación entre la Actuación y Evaluación en los juegos del rol y el Rendimiento Académico de los alumnos de la especialidad de periodismo de la EP de Comunicación Social de la UNMSM en el periodo 2017-II.

\section{Material y método}

La población y muestra de estudio estuvo conformada por 65 estudiantes de la especialidad de Periodismo de la EP de Comunicación Social de la UNMSM del octavo y décimo ciclo matriculados en el semestre académico 2017-II.
La investigación en lo funcional es de tipo descriptivo correlacional causal, porque se encontró la relación entre las variables Método didáctico de los Juego de Rol y el Rendimiento Académico. Y es descriptiva porque permitió describir las características de las variables.

El diseño adecuado para esta investigación es de tipo no experimental correlacional causal, cuantitativo y de corte transversal. Es transversal porque la recolección de los datos se realizó en un solo momento y en un tiempo único.

\section{Resultados}

Existe una correlación estadísticamente significativa entre los juegos de rol y el rendimiento académico de los alumnos de la especialidad de periodismo de la EP de Comunicación Social en el periodo 2017-II, dado que el valor de la significación bilateral (valor de $p$ ) es igual a $0,000(\mathrm{P}=0.000<0.05, \mathrm{r}=0.633)$, por lo que existe evidencia estadística para rechazar la hipótesis nula (Ho) y se concluye que existe una correlación directa y significativa entre el método didáctico de los juegos de rol y el rendimiento académico. Además, la correlación es directa y positiva, porque alcanza un nivel de 0.633 .

Se estableció una correlación estadísticamente significativa entre el rol del profesor y el rendimiento académico, dado que el valor de la significación bilateral (valor de $p)$ es igual a $0,000(\mathrm{P}=0.000<0.05$, $\mathrm{r}=0.524$ ), por lo que existe evidencia estadística para rechazar la hipótesis nula (Ho) y establecer que existe una correlación directa y significativa entre la dimensión rol del profesor y el rendimiento académico. Además, la correlación es directa y positiva, porque alcanza un nivel de 0.524 .

También se halló una correlación estadísticamente significativa entre la selección de participantes y el rendimiento académico, dado que el valor de la significación bilateral (valor de $p$ ) es igual a 0,000 $(\mathrm{P}=0.000<0.05, \mathrm{r}=0.585)$, por lo que existe evidencia estadística para rechazar la hipótesis nula $(\mathrm{Ho})$ y afirmar que existe una correlación directa y significativa entre la dimensión selección de los participantes y el rendimiento académico. Además, la correlación es directa y positiva, porque alcanza un nivel de 0.585 . 
Asimismo, se evidencia una correlación estadísticamente significativa entre las reglas de juego y el rendimiento académico, dado que el valor de la significación bilateral (valor de $p$ ) es igual a $0,000(\mathrm{P}=$ $0.000<0.05, r=0.644$ ), por lo que existe evidencia estadística para rechazar la hipótesis nula $(\mathrm{Ho})$ y se comprueba que hay una correlación directa y significativa entre la dimensión selección de los participantes y el rendimiento académico. Además, la correlación es directa y positiva, porque alcanza un nivel de 0.644 .

De la misma manera, se encontró una correlación estadísticamente significativa entre la actuación y evaluación y el rendimiento académico, dado que el valor de la significación bilateral (valor de $p$ ) es igual a $0,000(\mathrm{P}=0.000<0.05, \mathrm{r}=0.642)$, por lo que existe evidencia estadística para rechazar la hipótesis nula (Ho) y se concluye que existe una correlación directa y significativa entre la dimensión actuación y evaluación en los juegos rol y el rendimiento académico al $95 \%$ de confianza. Además, la correlación es directa y positiva, porque alcanza un nivel de 0.642 .

\section{Discusión}

Los resultados de la investigación evidencian que el método didáctico de los juegos de rol tiene una relación directa y significativa $(r=0.633)$ con el rendimiento académico de los estudiantes de la especialidad de periodismo de la EP de Comunicación Social de la UNMSM. Si bien es cierto que hay correlación entre las variables mencionadas, la correlación no es perfecta $(r=1)$. En ese sentido, podemos afirmar que se puede mejorar la aplicación del método didáctico de los juegos de rol en la especialidad de periodismo de la EP de Comunicación Social de la UNMSM con el fin de incidir favorablemente en el rendimiento académico de los estudiantes y en su desarrollo como futuros profesionales del periodismo.

Amézquita y Moreno (2001, pp. 65-66) sostienen que para la construcción de un juego de rol educativo es necesario el análisis de contenido y semiótico de los conocimientos que se desean compartir con los estudiantes. En el caso de los juegos de rol en nuestro medio podemos observar qué es necesario una sistematización de los métodos didácticos en los cursos de la especialidad de periodismo para mejorar la formación profesional.

Joyce y Weil (2009) sostienen que el modelo de los juegos de rol «sumamente versátil y aplicable a varios objetivos educacionales importantes» (p. 101). Afirman, además, que es un vehículo para desarrollar el contenido de las enseńanzas en la medida en que los estudiantes disfruten de la acción y la actuación.

De manera similar, se encontró una relación estadísticamente significativa de $\mathrm{r}=-0.524$ en un nivel regular y directo entre el rol del profesor y el rendimiento académico al $95 \%$ de confianza. Este valor indica una correlación positiva entre el papel del profesor en el método didáctico de los juegos de rol periodísticos y el rendimiento académico de los estudiantes de la especialidad de periodismo de la EP de Comunicación Social de la UNMSM en el periodo 2017-II.

Joyce y Weil (2009, p. 94) afirman que el profesor tiene la misión de presentar un problema social de interés público que debe captar el interés de los alumnos mediante un ejemplo de la vida cotidiana en la fase preparatoria de los juegos de rol con el fin de sensibilizar a los estudiantes con el tema y puedan exponer todas sus ideas, sentimientos y conductas con libertad.

En este caso, el docente puede usar como ejemplo, la reciente detección de una organización criminal dirigida por jueces corruptos dedicados al tráfico de influencias para favorecer a violadores y narcotraficantes. La actividad de estos sujetos conocidos policial y judicialmente como «Los Cuellos Blancos del Puerto» ha generado un escándalo e indignación en la ciudadanía gracias a la difusión de audios comprometedores por medios independientes como IDL-Reporteros.

Los mismos autores recomiendan que el docente y los alumnos pueden enfrascarse en la presentación de una serie de ejemplos del tema en cuestión en base a todo tipo de fuentes: desde las informaciones proporcionadas por los medios de comunicación hasta las de tipo personal. La finalidad es formular preguntas que promuevan la reflexión y la predicción del desenlace por parte de los estudiantes.

Se encontró una relación estadísticamente significativa de $r=-0.585$ entre la selección de los participantes en el juego de rol y el rendimiento académico al 95\% de confianza en la especialidad de periodismo 
de la EP de Comunicación Social de la UNMSM en el periodo 2017-II. Este resultado indica un nivel regular y directo de correlación entre la selección de participantes (identificación con los roles, elección de los líderes, sanción democrática de los infractores y arbitrio constante en clase) y el rendimiento académico de los alumnos de en la especialidad de periodismo de la EP de Comunicación Social de la UNMSM en el periodo 2017-II.

Joyce y Weil (2009) afirman que existen varios criterios para seleccionar a un alumno para un rol en especial y mencionan a continuación lo siguiente:

Los roles pueden asignarse a quienes parecen tan involucrados en el problema que se identifican con un rol específico; a los que manifiestan una actitud que necesita ser analizada y a quienes deben aprender a identificarse con el rol o ponerse en la posición de otra persona. (p. 96)

Flórez, R. (1994), en este punto, afirma que:

La enseñanza constructivista ostenta como principio partir de la estructura mental del alumno, y ello implica reconocer no solo sus ideas y prejuicios sobre el tema de la clase, sino inclusive reconocer el nivel de pensamiento lógico que posee el alumno para propiciarle experiencias que promuevan sus habilidades de pensamiento en el campo de los fenómenos objeto de la ciencia particular de enseñanza. (p.246)

En ese sentido ańade que:

Se trata de que el educador esboce las experiencias educativas pertinentes de modo que partiendo de lo que el alumno ya sabe y es capaz de operar cautive su curiosidad intelectual con un buen interrogante, y le suministre las señales apenas suficientes como acícate y orientación para que el alumno se lance por su cuenta propia a la aventura del pensamiento, hasta elevarse por encima sí mismo a la conquista de nuevos horizontes. (p. 246)

Se encontró una relación estadísticamente significativa de $\mathrm{r}=0.644$ entre las reglas de los juegos de rol y el rendimiento académico al 95\% de confianza en la especialidad de periodismo de la EP de Comunicación Social de la UNMSM en el periodo 2017-II. Este valor indica un nivel directo de corre- lación entre las reglas de los juegos de rol (técnicas de redacción, pautas de conducta, uso de apuntes y grabadoras, elaboración de la agenda periodística y revisión de los avances) y el rendimiento académico de los alumnos de la especialidad de periodismo de la EP de Comunicación Social de la UNMSM en el periodo 2017-II.

Las reglas de los juegos de rol educativos tienen que ver con los contenidos que se quieren transmitir. En ese caso, Amézquita y Moreno (2001) sostienen que el juego:

Es conducido por un director, participante del juego que da orden a la actuación de los personajes, resuelve los conflictos entre los jugadores y, sobre todo, conoce a fondo las reglas, la historia, el ambiente, el terreno y los personajes con el fin de dar coherencia, equidad y diversión a un juego de rol. En ocasiones, el director es quien también diseña el ambiente y la historia. (p. 34)

En el caso de los cursos de la especialidad de periodismo son los profesores quienes organizan los contenidos conceptuales y encargan las comisiones periodística, sistematiza las pautas de conducta y revisa constantemente los informes semanales elaborados por los alumnos. De la misma manera, vela por la cohesión de los grupos.

Se encontró una relación estadísticamente significativa de $\mathrm{r}=-0.642$ entre la actuación y evaluación en los juegos de rol y el rendimiento académico al $95 \%$ de confianza en la especialidad de periodismo de la EP de Comunicación Social de la UNMSM en el periodo 2017-II. Este valor indica un nivel moderado y directo de correlación entre la actuación y evaluación en los juegos de rol (evaluación de datos, verificación de datos, redacción periodística, creatividad estilística, y evaluación y autoevaluación) y el rendimiento académico de los alumnos de la especialidad de periodismo de la EP de Comunicación Social de la UNMSM en el periodo 2017-II.

La actuación y evaluación es el producto del desempeño de los alumnos luego de asumir su rol de periodistas en relación con los hechos de interés público que tienen un impacto en la sociedad y son vigentes. Joyce y Weil (2009) afirman que es deber del docente configurar el análisis de los resultados de los juegos de 
rol, para que los alumnos puedan generalizar sobre la forma de abordar y solucionar situaciones problemáticas en el ejercicio del periodismo.

\section{Conclusiones}

Los hallazgos de la investigación dan cuenta de la existencia de una relación directa y significativa entre el método didáctico de los juegos de rol y el rendimiento académico de los alumnos de la especialidad de periodismo de la EP de Comunicación Social en el periodo 2017-II ( $r=0.633$ ).

Se evidencia una correlación significativa en un nivel directo entre el rol del profesor y el rendimiento académico de los alumnos de la especialidad de periodismo de la EP de Comunicación Social en el periodo 2017-II ( $\mathrm{r}=0.524)$.

Se observó una correlación significativa en un nivel directo entre la selección de los participantes en el juego de rol y el rendimiento académico de los alumnos de la especialidad de periodismo de la EP de Comunicación Social en el periodo 2017-II $(\mathrm{r}=0.585)$.

Los resultados de la investigación demuestran la existencia de una correlación significativa en una relación directa entre las reglas de los juegos de rol y el rendimiento académico de los alumnos de la especialidad de periodismo de la EP de Comunicación Social en el periodo 2017-II ( $\mathrm{r}=0.644$ ).

Se encontró una correlación significativa y directa entre la actuación y evaluación en los juegos de rol y el rendimiento académico de los alumnos de la especialidad de periodismo de la EP de Comunicación Social en el periodo 2017-II ( $\mathrm{r}=0.642)$.

\section{Referencias bibliográficas}

Fuentes impresas:

Amézquita, I. y Moreno, M. (2001). La odisea liberadora de los juegos de rol. Jalisco, México: Instituto Tecnológico y de Estudios Superiores de Occidente, Universidad Jesuita de Guadalajara.

Birkenbihl, M. (2008). Formación de formadores. Manual práctico para educadores y profesores, con 21 juegos de rol y estudio de casos. Madrid, Espańa: Ediciones Paraninfo-universidad.

Edó, C. (2009). Periodismo informativo e interpretativo. El impacto de Internet en la noticia, las fuentes y los géneros. México, México D.F.: Alfaomega Grupo Editor S.A.

Flórez, R. (1994). Hacia una pedagogía del conocimiento. Bogotá, Colombia: McGraw-Hill, Interamericana S.A.

Joyce, B. y WeIL, M. (2009). Modelos de enseñanza (3a ed.). Barcelona, España: Editorial Gedisa.

Kapuscinski, R. (2002). Los cínicos no sirven para este oficio. Sobre el buen periodismo. Barcelona, España: Anagrama.

VAN Dijk, T. A. (1990). La noticia como discurso. España, Barcelona: Ediciones Paidós Ibérica.

VAn Dijk, T. A. (1997). Racismo y análisis crítico de los medios. España, Barcelona: Ediciones Paidós Ibérica.

Fuentes de tesis:

CÁCERES, N. J. (2012). Juegos de roles como estrategia motivadora en el aprendizaje por competencia en los cadetes de la Escuela Militar de Chorrillos. Lima, Perú.

Forero, D, y LoAiza (2013). El juego de rol: una estrategia para el aprendizaje del inglés de los estudiantes de grado transición de un colegio privado de Bogotá, Colombia.

GaEte-Quezada, R. (2011). El juego de roles como estrategia de evaluación de aprendizajes universitarios de la Universidad de Antofagasta, Chile.

Giraldo, Y. (2014). Juegos de rol para mejorar la producción y comprensión oral en estudiantes de francés II, Colombia.

MarTínez, José (2009). Influencia del rol-playing en el proceso de enseñanza-aprendizaje de enfermería. Tesis doctoral, Universidad de Alicante. Espańa.

Vera-Mostacero, N. (2007). Influencia del juego de roles para mejorar la comprensión y producción oral en el idioma inglés de los alumnos del quinto grado de educación secundaria del colegio particular adventista José de San Martin de Trujillo. Perú. 